University of Nebraska - Lincoln

DigitalCommons@University of Nebraska - Lincoln

Educational Psychology Papers and

Publications

Educational Psychology, Department of

November 1994

\title{
Implementing Scientific Practices Through Case Studies: Examples Using Home-School Interventions and Consultation
}

JoAnn Galloway

Jordan (Utah) School District

Susan M. Sheridan

University of Nebraska-Lincoln, ssheridan2@unl.edu

Follow this and additional works at: https://digitalcommons.unl.edu/edpsychpapers

Part of the Educational Psychology Commons

Galloway, JoAnn and Sheridan, Susan M., "Implementing Scientific Practices Through Case Studies: Examples Using Home-School Interventions and Consultation" (1994). Educational Psychology Papers and Publications. 30.

https://digitalcommons.unl.edu/edpsychpapers/30

This Article is brought to you for free and open access by the Educational Psychology, Department of at DigitalCommons@University of Nebraska - Lincoln. It has been accepted for inclusion in Educational Psychology Papers and Publications by an authorized administrator of DigitalCommons@University of Nebraska - Lincoln. 


\title{
Implementing Scientific Practices Through Case Studies: Examples Using Home-School Interventions and Consultation
}

\author{
JoAnn Galloway, Jordan (Utah) School District
}

\author{
Susan M. Sheridan, University of Utah
}

\begin{abstract}
Integrating scientific methods into school psychological practice is an essential tenet of effective service. In this article, we present two related examples that demonstrate methods by which to conduct high-quality intervention and consultation case studies with integrity. The present case studies utilized home note and consultation-based interventions to improve task completion and accuracy in mathematics for primary grade students who had demonstrated performance difficulties despite adequate intellectual ability and academic skills. Two sets of case studies were conducted. One involved the use of a home-school note implemented by parents and teachers in conjunction with a self-instruction manual. The second set of case studies used the home note and manual instituted in the context of behavioral consultation with parents and teachers. Both interventions (home-note-only and home note with consultation) increased math completion and accuracy in underachievers. Levels of performance were stable and differences between baseline and treatment conditions were statistically significant for consultation students only. Treatment integrity, maintenance of treatment gains, and consumer acceptability were also stronger in the consultation case studies. The implications for practitioners and future research are explored.
\end{abstract}

Keywords: Home-school intervention, Conjoint consultation, Academic underachievement, Home note, Scientist-practitioner.

The integration of science and practice is increasingly recognized as essential to ethical service delivery of school psychology services. There are several methods of enhancing the science-practice relationship, such as remaining abreast in current research findings and conducting empirical investigations of professional practices. Conducting service-related research in the schools, however, is challenging. The purpose of this article is to demonstrate how two scientifically based interventions (i.e., home-school notes and conjoint behavioral consulta- 
tion) can be applied and evaluated by a school psychologist practitioner in the remediation of academic performance deficits. First, we will review considerations in the designing of case studies for increasing their ability to suggest empirical and functional relationships between interventions and behavior change. We will then review relevant literature regarding the intervention procedures to support their choice in this set of cases. Specific case study methods used by the school psychologist will be described, along with results gleaned from objective measures.

\section{APPLIED RESEARCH IN SCHOOL PSYCHOLOGY PRACTICE}

In school-based research, the necessity of employing stringent experimental controls and carefully manipulating independent variables is often compromised by practical and logistical realities. Problems with students' absences, school schedules, changing routines, and other uncontrollable systemic variables present difficulties for practitioners desiring to conduct school-based research. Even single-subject experimental methods such as multiple-baseline designs are difficult in some intervention situations, given the need for strict subject selection criteria and systematic treatment programming. These challenges are compounded by the fact that the various additional job demands of school psychologists, such as evaluation and crisis intervention, often require that research and other accountability practices take on secondary importance.

There is clearly a need for school psychology practitioners to conduct research on their services to clients. A number of single-subject experimental and preexperimental case study designs are available to practitioners conducting research in the schools. Whereas rigorous experimental designs such as the multiple-baseline or alternating treatment designs are preferred because they pose fewer threats to internal validity, even carefully controlled case study designs such as the A-B design can suggest functional relationships between interventions and behavior change. Such a functional relationship can be corroborated with multiple measures, replication across subjects, and follow-up evaluation (that is, assessment after a treatment is terminated)

Data should be collected to assess outcomes, evaluate impact, and provide documentation for accountability. Furthermore, to understand the breadth of impact, data should be collected across a number of dependent measures. Variables such as intervention effectiveness, treatment acceptability, integrity of services, and social validity should be investigated.

Several methods for increasing the strength of case studies can be utilized. First, careful articulation of the target behavior, assessment methods, and intervention procedures are important. Operational definitions that are concrete, specific, and objective help clarify central aspects of the case studies. Using repeated observations and replicating the design over several cases can support conclusions made in intervention work. Other procedures to strengthen intervention case studies and minimize various threats to internal validity include (a) the use of objective measures, (b) active control and manipulation of the independent variable, (c) standardization of assessment and treatment, (d) checks on treatment integrity, (e) social validity assessment, (f) a planned design with follow-up evaluation, (g) time series analyses, and (h) replication (Kratochwill, 1985). These are described in the context of school-based case studies below.

\section{Assessment Considerations}

Careful articulation of target behaviors and assessment procedures are central to case studies. In general, multiple assessment methods are preferred because they can provide information on treatment impact across various aspects of a student's functioning. Objective procedures should be used, including objective behavioral checklists (as opposed to projective or subjective assessment methods) and clearly operationalized observational techniques. Repeated observations are important to monitor the effects of an intervention on a target behavior. This requires that observational measures be taken several times during baseline, treatment, and follow-up phases. If possible, a classroom aide or teacher's assistant can be solicited to collect observational data in a repeated fashion. The observational task can be simplified further by using existing data as the dependent measure. For example, the number of items completed or percentage correct on a homework assignment can be collected easily over time.

\section{Intervention Considerations}

Planned control and manipulation of the intervention (i.e., the independent variable), rather than passive observations of events can assist practitioners in deriving inferences from case study investigations. Specification of the point at which the intervention will be implemented provides practitioners with more control over extraneous variables. Relatedly, the intervention procedures should be standardized (i.e., clearly specified) to allow for replication with other students. Several standardized interventions with manuals are available for various target problems, such as social skills (e.g., McGinnis \& Goldstein, 1984) and homework (e.g., Olympia, Jenson, \& Neville, 1990). When the intervention has demonstrated effectiveness with other subjects or target behaviors, similar case study results can be interpreted more directly.

Checks on treatment integrity are important to ensure that the intervention is being implemented in a manner consistent with its design. In other words, consultants cannot simply assume that a teacher, parent, or student is carrying out a treatment correctly (i.e., as intended). Repeated checks on the implementation of treatments are necessary and can be made in several relatively simple and straightforward ways. For example, direct observations of the treatment agent (e.g., teacher) can be conducted during treatment sessions. Likewise, checklists 
can be devised that outline the steps of the intervention procedure for self-monitoring purposes. Other various procedures for assessing treatment integrity are presented in Gresham (1989).

\section{Social Validation}

Social validity refers to the clinical meaningfulness of treatment changes (Kazdin, 1977). The types of social validation most relevant to case studies are social comparison and subjective evaluation. When social comparisons are conducted, the behavior of a subject before and after treatment can be compared with that of peers who do not demonstrate the problem behaviors. This can be accomplished easily by direct observations or rating scale data. A clinically important change would be noted if a target student's behaviors were brought to a level similar to those of nondeviant peers following treatment. Subjective evaluations are collected to assess the degree to which significant others (e.g., parents and teachers) perceive treatment changes as meaningful. For example, responses to selected items on behavior-specific rating scales can be used to assess adults' perceptions of a student's behavioral changes.

Treatment acceptability is a special form of social validity. The importance of treatment acceptability is based on the assumption that treatment agents will be more willing to implement an intervention with integrity if it is acceptable to them. Standard forms assessing treatment acceptability are available (Kazdin, French, \& Sherik, 1981; Von Brock \& Elliott, 1987), and this variable is receiving a great deal of attention in applied research.

\section{Replication}

Practitioners may find occasion to implement identical treatments (e. g., social skills training) with several students. In these situations, it is possible to conduct identical A-B case studies for purposes of replicating the procedures and assessing the effects across students. When several students show positive and immediate responsiveness to the same treatment, more confidence can be placed in the functional relationship between the intervention and students' behavioral changes. Replication across individual students at different points in time is preferred (Kratochwill, 1985).

With these considerations in mind, two sets of case studies were designed to evaluate the effects of treatments aimed at remediating students' academic performance deficits. This target was selected in view of the challenge it poses to those responsible for their education. Specifically, students who are intellectually and academically competent but who have difficulty in getting organized to work, sustaining attention and effort over time, and completing school tasks efficiently and accurately cause concern for teachers and parents.

The first stage of applied research is being an informed consumer: that is, remaining abreast of current research and evaluating the application of empirical findings as they relate to respective professional settings. Therefore, extensive literature searches were conducted on procedures that were both empirically validated and practically relevant. The use of home-school notes emerged as an appropriate strategy meeting both of these criteria. Methods for increasing the effectiveness of home-school notes were explored, and consultation services with parents and teachers appeared particularly appealing, given their emphasis on collaborative problem solving and data-based outcomes.

\section{HOME-SCHOOL NOTES AND CONJOINT BEHAVIORAL CONSULTATION}

Home-school notes have been used as a means of remediating academic and behavioral difficulties at school. Home-school notes usually involve the implementation of home rewards and sanctions based on the teacher's report of academic and social behaviors at school. They require little teacher time in comparison with other classroom behavioral interventions, they provide a vehicle for regular communication between home and school, and they allow for the provision of a richer menu of reinforcers than is generally available within the confines of the classroom. Their usefulness has been demonstrated with behavioral (Budd, Leibowitz, Riner, Mindell, \& Goldfarb, 1981; Lahey et al., 1977) and academic performance problems (Blechman, Kotanchik, \& Taylor, 1981; Blechman, Taylor, \& Schrader, 1981; Schumaker, Hovell, \& Sherman, 1977).

Although some form of consultation is generally implied in the implementation of home-school notes (Fairchild, 1976), procedures and participant roles have rarely been discussed (Sheridan \& Kratochwill, 1992). Children with significant behavioral or academic problems may require a consultant to provide parents and teachers with detailed training to maximize the effectiveness of the home note procedure (Kelley \& Carper, 1988). A behavioral consultation format may serve this purpose by providing a framework for active involvement of participants in the problem-solving process.

Behavioral consultation provides an empirically sound framework for providing indirect services to a client (e.g., student) through a consultee (e.g., parent, teacher). The seminal work of Bergan and Kratochwill (Bergan \& Kratochwill, 1990; Kratochwill \& Bergan, 1990) provides a systematic structure for the practice of behavioral consultation through a four-stage process (problem identification, problem analysis, treatment implementation, treatment evaluation), operationalized by a series of standardized behavioral interviews.

Conjoint behavioral consultation (CBC) is similar to behavioral consultation in its stages and objectives. In $\mathrm{CBC}$, however, behaviors are assessed and treated across both home and school environments to maximize the desired effects. Specifically, parents and teachers work together as co-consultees (a) to identify the problems(s) to be targeted in consultation, (b) to identify factors across settings that might influence the attainment of the problem solution, (c) to design and im- 
plement a treatment plan to be implemented at home and school, and (d) to evaluate its success across settings, making modifications as needed (Sheridan \& Kratochwill, 1992).

Research on the effectiveness of CBC is accumulating. Sheridan, Kratochwill, and Elliott (1990) found that socially withdrawn subjects demonstrated increased social initiations toward peers in both the home and the school as a function of behavior programming implemented in the context of $\mathrm{CBC}$. When consultation was conducted with teachers only, however, behavioral changes were noted at school with no generalization to the home. In a single-case study, Sheridan and Colton (1994) demonstrated immediate and long-term behavioral changes in a kindergarten student with irrational fears when CBC services were provided. In both studies, the authors reported a need to investigate the efficacy of the model with various target behaviors, such as academic performance deficits.

It is conceivable that the use of a structured parent-teacher consultation framework may be useful in the remediation of academic performance problems, particularly for students whose academic problems have proven resistant to other methods. In the remainder of this article, we describe a school psychologist's intervention efforts to improve task completion and accuracy in mathematics for primary-grade students who demonstrated performance difficulties despite adequate intellectual ability and academic achievement. For three cases, a home note strategy, in which parents were provided specific techniques for reinforcing school achievement at home, was used to increase the academic productivity of academically underachieving students with a history of previous intervention failure. For three similar cases, the home note strategy was used in the context of a CBC model involving parents, teachers, and students.

\section{GENERAL METHODS FOR CASE STUDIES}

Two sets of case studies were conducted, each with the intervention procedures replicated over three students. In the first set of cases (Students 1-3), a home note and self-instruction manual was used in the treatment of academically underachieving students (i.e., students with performance deficits in math completion and accuracy, despite adequate ability and previous intervention attempts). In the second set of cases (Students 4-6), the same home note and manual were used; however, the intervention was implemented in the context of CBC services to parents, teachers, and students.

\section{Setting}

The setting for the case studies was a suburban public elementary school that serves primarily middle-class and upper-middle-class families on a year-round basis. The year-round schedule consists of rotating tracks with students "on track" (in school) for approximately 9 weeks and "off track" (out of school) for approximately 3 weeks. All experimental procedures were conducted in elementary school classrooms. Consultation sessions were held in the school psychologist's office.

\section{Target Students}

Six students (two boys and four girls) from the primary grades (Grades 13 ) and their parents and teachers participated in the case studies. Because the intent of the studies was to evaluate the effectiveness of motivational interventions, students were chosen who demonstrated the necessary math skills to perform at grade level. Task analyses revealed that when the subjects completed their work, it tended to be accurate. However, they often failed to complete assigned tasks, so their accuracy scores reflected the amount of work completed. In sum, target students (a) demonstrated an inconsistent performance pattern; (b) consistently failed to complete their assigned work in mathematics; and (c) often achieved math accuracy scores below $70 \%$, due primarily to lack of work completion.

Potential target students who appeared to meet the criteria of adequate math skills but who demonstrated poor or inconsistent daily performance in math were nominated by their teachers. Written permission to assess academic skills was obtained from parents. Students were screened with a brief intelligence test, the Kaufman Brief Intelligence Test (Kaufman \& Kaufman, 1990), and four subtests (Letter-Word Identification, Passage Comprehension, Calculation, Applied Problems) from the achievement section of the Woodcock-Johnson Psychoeducational Battery-Revised (Woodcock \& Mather, 1990). Only students who scored within the average range (i.e., standard scores of 85 or above) on all measures were considered for the studies. This criterion was necessary to rule out the presence of learning disabilities, which could confound results and make interpretations difficult.

\section{Procedures}

Two separate sets of case studies were conducted. The first consisted of a home note and an accompanying manual aimed at assisting parents in reinforcing their children for good school performance. The second involved the same home note procedure implemented in the context of $\mathrm{CBC}$. The students were assigned to the conditions randomly.

Home Note Procedures. The home note was a single-page form on which teachers recorded individual students' daily math scores, four task completion behaviors (i.e., paper and pencil ready; start working right away; work until the job is finished; and hand the work in) and work completion percentages. A selfinstructional home note manual was prepared to be used in conjunction with the 
notes. The manual was a six-page document that provided (a) empirical support for the effectiveness of home-school notes, (b) general procedures for setting up a home note, (c) suggestions for potential high-interest reinforcers, and (d) procedures for handling problems that might occur with the home note. Parents were encouraged to provide to their child home rewards contingent upon the day's math performance. The criteria for earning home rewards was completion of assigned math tasks within the allocated academic period with at least $80 \%$ accuracy.

CBC Procedures. Three parents and teachers were involved in CBC with the school psychologist in a program involving four stages operationalized through a series of standardized behavioral interviews. Structured forms (Sheridan, Kratochwill, \& Bergan, in press) were used by the school psychologist to conduct Problem Identification (PII), Problem Analysis (PAI), and Treatment Evaluation Interviews (TEI). The time required for the interviews ranged from 10 to $38 \mathrm{~min}$ utes. They were conducted prior to baseline (PII), prior to the implementation of the home note intervention (PAI), and at the end of treatment (TEI). Approximately 5 days elapsed between the PIIs and PAIs and there were approximately 26 days between the PAIs and TEIs. In the present cases, consultees included the teacher and parent in the problem identification stage. In the problem analysis and treatment evaluation stages, the student was also involved in the interviews.

In these case studies, the problem behavior and treatment program were identified prior to consultation. This was necessary to ensure that identical procedures could be replicated across students, thus strengthening the A-B design (Kratochwill, 1985). While this may seem contrary to basic consultation principles, it can be recalled that students whose teachers had concerns about their math performance despite adequate abilities were referred for inclusion. Therefore, teachers had recognized the problem as a priority for intervention. Furthermore, although the general home note procedure was imposed as the "treatment of choice" for all cases, various aspects of the procedures were individualized across consultation students. For example, during PAIs various personal and environmental factors that contributed to the performance of the individual student were identified and integrated into the program. In some cases, distractions in the classroom were removed. In another case, a pattern in the student's errors was detected, which suggested the need for continuous checks on his performance. The selection and delivery of home-based reinforcers were also individualized on the basis of information learned in the consultation interviews. In addition, the interviews provided an opportunity to discuss early intervention procedures. This became particularly important in the case of one student, whose performance dropped dramatically during the early days of the intervention due to a misunderstanding between the child and her mother regarding the criteria for earning the home reward.

\section{Outcome Measures}

Several outcome measures were used to investigate the efficacy, social validity, acceptability, and integrity of the home note and consultation interventions. Math performance was analyzed in terms of assignment completion and accuracy. Social validation was evaluated by social comparison and subjective evaluation methods. Treatment acceptability was assessed by parent, teacher, and student ratings. Treatment integrity was assessed through self-monitoring, observations, and anecdotal interviews.

Math Performance. Students' daily classroom math assignments were collected and scored for completion and accuracy. These are considered meaningful, curriculum-based measures, as they assess the curriculum presented in classroom instruction (Salvia \& Hughes, 1990). Data on the percentage of math work completed and daily math scores were collected for each subject across baseline, treatment, and follow-up conditions. Daily math scores were calculated in a manner similar to that used by classroom teachers (i.e., the number of math problems completed correctly divided by number of problems assigned multiplied by 100). Completion data were calculated as the number of problems completed divided by the number of problems assigned multiplied by 100 . All math scores were calculated daily at the end of the time allotted for the class to complete the math assignment. They were also calculated for a 5-day follow-up period 7 weeks after termination of the intervention.

Treatment Acceptability. The Behavior Intervention Rating Scale (BIRS) (Von Brock \& Elliott, 1987) was used to assess the degree to which parents and teachers found the treatments acceptable. The BIRS consists of 24 items rated on a 6-point Likert-type scale. Evidence for the reliability of the BIRS is available (Von Brock \& Elliott, 1987). A recent factor analysis study (Elliott \& Van Brock Treuting, 1991) identified three factors (Acceptability, Effectiveness, and Time to Effect) with moderate to strong correlations among them.

The target students rated their perceptions of the acceptability of treatments by responding to the Children's Intervention Rating Profile (CIRP) (Turco \& Elliott, 1986). This scale consists of six items pertaining to the fairness, acceptability, and potential side effects of interventions for particular problems. Items are rated on a 6-point Likert-type scale ranging from I agree to I do not agree. Concurrent and predictive validity data are currently lacking for the CIRP however, it has been used widely with children across a number of studies with very infrequent failures to respond to an item and very few response sets (E1liott, 1986). The scale was read to students as needed to insure comprehension of the item content. 
Social Validity. Two forms of social validity (i.e., social comparison and subjective evaluation) were used to support findings in the case studies. Social comparison procedures were used to compare the behaviors of target students with those of matched peers whose behaviors had not been considered problematic (Kazdin, 1977). Specifically, the target students' average math scores recorded by teachers during intervention were compared with the same data for nonreferred peers matched on sex, classroom, and approximate intellectual and academic achievement levels.

Selected items from the BIRS were analyzed for purposes of subjective evaluation. Global ratings of the degree of improvement in target behaviors as a result of the intervention were provided by both parents and teachers through their responses on two BIRS items (i.e., "The intervention proved effective in changing the child's problem behavior"; and "The intervention quickly improved the child's behavior"). In addition, one open-ended question was provided for teachers (i.e., "To what degree did the child's performance in math, as a result of the intervention, match that of children in your class whose math performance is adequate?").

Treatment Integrity. Self-monitoring data were collected to assess the integrity (i.e., adherence to treatment components) of the behavioral program by parents and teachers (Gresham, 1989). Specifically, daily checklists were developed for the teachers and parents. The teacher checklist included such items as checking for the presence of the note, scoring and recording daily math scores and completion percentages, and ensuring that the student took the note home at the end of the day. The parent checklist included three items, which were included at the bottom of the home note for ease and efficiency: "Check the home note, praise child for good performance, and provide agreed-upon reward when earned."

For three cases, the integrity of the CBC procedures was assessed. Specifically, all $\mathrm{CBC}$ interviews were audiotaped and rated by independent coders. Coders were trained to identify specific criteria for each interview, using behavioral consultation objectives checklists from previous research (Kratochwill, Sheridan, Rotto, \& Salmon, 1991; Kratochwill, VanSomeren, \& Sheridan, 1989; Sheridan, 1992). The percentages of interobserver agreement in training sessions were $94 \%$ for PIIs, $94 \%$ for PAIs, and $92 \%$ for TEIs. One coder rated all of the interviews, and reliability was determined by a second rater who rated a random sample of $55 \%$ of the interviews. The sample included all interview types (i.e., two PIIs, one PAI, and two TEIs), and represented interviews concerning all three CBC students. A kappa coefficient was computed to assess the proportion of coder agreement corrected for chance.

Anecdotal interviews were conducted to assess informally parents' implementation of the program. Specifically, an independent interviewer contacted each parent by telephone three times during the course of the treatment to ask parents (a) about their child's performance in math that day, (b) whether home rewards were provided, (c) what types of rewards were used, (d) whether any aversive consequences were employed if children failed to meet the performance criteria, and (e) whether the parent had had contact with the teacher during the week.

\section{Case Study Design Considerations}

An A-B design with replications was used to analyze the effects of the interventions (Harris \& Jenson, 1985). A multiple-baseline across-subjects design was originally planned to control various threats to internal validity; however, a number of factors precluded its use. The primary difficulty was related to the school's year-round schedule. Several problems were encountered in the effort to coordinate interviews and interventions while students were continuously going on track and off track. Timing the consultation interviews and interventions, coordinating parent and teacher schedules, and maintaining the methodological demands of multiple-baseline designs within the year-round framework became logistically impossible. The A-B design with replication is considered valid and of particular usefulness in dealing with the complexities of natural settings (Harris \& Jenson, 1985).

The baseline condition ranged from 9 to 14 days. Baseline consisted of simple monitoring of students' math completion and accuracy by teachers with no special contingencies in effect. In most cases, baseline data should be stable before an intervention phase is initiated. With these students, however, variability in performance was one criterion for inclusion. Given that stability could not be achieved, baseline was extended to gain a better understanding of pretreatment performance. Once a relatively clear picture of pretreatment performance was obtained, consultation and/or the home-note treatment was implemented as soon as possible.

Treatments were administered for periods ranging from 14 to 17 days. Treatment for three students involved physically handing the home note to the parents and teachers, with introductory phone calls to parents to instruct them on starting dates and general procedures. For the remaining three students, treatment consisted of the same home note intervention, implemented in the context of CBC Specifically, the parents and teachers met with the school psychologist for joint decision making about home contingencies and ongoing troubleshooting regarding treatment implementation and effectiveness. Approximately 7 weeks following the completion of treatment, five follow-up probes were conducted for all the subjects to assess treatment maintenance.

The data were analyzed visually, descriptively, and statistically. Changes in level were analyzed by comparing average amounts of work completion and accuracy across baseline, treatment, and follow-up conditions for each student. Variability was analyzed by methods recommended by Tawney and Gast (1984). By these criteria, data are considered stable when $80 \%-90 \%$ of data points fall 
within a $15 \%$ range of the mean. Checklist and rating scale data were analyzed descriptively.

Statistically, changes in level and slope between baseline and treatment conditions were analyzed by interrupted time series analysis (DMITSA) procedures (Crosbie \& Sharpley, 1991). DMITSA develops a model of the dependency (autocorrelation) in a time series. Significant autocorrelation (dependence among data points) was found in this data series, so the use of DMITSA (which removes autocorrelation so that statistical tests can be employed) was appropriate. ${ }^{1}$ The interrupted time series analysis was used to determine whether a significant change was evidenced between baseline and treatment phases. DMITSA provides an F-value and indicates the probability that such a value would occur by chance.

\section{RESULTS}

\section{Math Accuracy}

Math accuracy scores for all students are presented in Table 1. Brief descriptions of the findings follow.

Home Note Intervention. All subjects who received the home note intervention only (without $\mathrm{CBC}$ ) increased their math scores from baseline to intervention, with gains in mean scores of $84 \%, 20 \%$, and $28 \%$ for Students 1,2 , and 3 , respectively. Students 1 and 2 improved their math performance from failing or nearly failing to a range comparable with that of peers who were considered subjectively by their teachers to be good math students. Student 3 did not raise her performance to an acceptable level. In this case, however, the program was carried out inconsistently, the home notes having been returned to school on only $38 \%$ of treatment days.

Math accuracy and completion data are presented graphically in Figure 1. Visual inspection indicates that baseline data for students who received the homenote-only intervention were variable (which is consistent with selection criteria), and continued to be variable during treatment. In fact, during treatment only $27 \%-36 \%$ of the data points fell within $15 \%$ of the mean scores for all three students. In addition, the intervention did not result in immediate changes in math performance for any students who received only the home note intervention. Finally, there is considerable overlap in data points between baseline and treatment for all three of these students. The variability also is apparent in the large standard deviations in baseline that increased even further during treatment.

${ }^{1}$ According to Crosbie and Sharpley (1991), interrupted time series analyses (ITSA) "remove autocorrelation from a data series so that statistical tests can be legitimately employed to determine whether there is a significant change (i.e., not due to chance) from Phase 1 to Phase 2" (p. 12). In effect, ITSA removes autocorrelation from the data and then examines the residuals.
Table 1 Math Accuracy Scores Across All Phases and Students

\begin{tabular}{|c|c|c|c|c|}
\hline \multicolumn{2}{|c|}{ Student } & Baseline & Treatment & Follow-up \\
\hline \multicolumn{5}{|c|}{ Home-note-only group } \\
\hline \multirow{4}{*}{1} & $M$ & 43.3 & 79.7 & 77.6 \\
\hline & $S D$ & 17.6 & 24.7 & 30.2 \\
\hline & Median & 44.0 & 91.5 & 90.0 \\
\hline & Range & $18-70$ & $25-100$ & $24-100$ \\
\hline \multirow[t]{4}{*}{2} & $M$ & 69.1 & 82.9 & 73.4 \\
\hline & $S D$ & 22.1 & 24.9 & 30.7 \\
\hline & Median & 70.0 & 94.0 & 76.0 \\
\hline & Range & $39-100$ & $26-100$ & $29-100$ \\
\hline \multirow[t]{4}{*}{3} & $M$ & 52.4 & 67.2 & 78.4 \\
\hline & $S D$ & 26.4 & 32.1 & 27.4 \\
\hline & Median & 54.0 & 75.0 & 95.0 \\
\hline & Range & $22-97$ & $4-100$ & $47-100$ \\
\hline \multicolumn{5}{|c|}{ CBC group } \\
\hline \multirow[t]{4}{*}{4} & $M$ & 36.5 & 89.1 & 59.0 \\
\hline & $S D$ & 19.1 & 10.3 & 8.5 \\
\hline & Median & 36.0 & 90.0 & 59.0 \\
\hline & Range & $0-66$ & $66-100$ & $53-65$ \\
\hline \multirow[t]{4}{*}{5} & $M$ & 34.8 & 86.5 & 96.2 \\
\hline & $S D$ & 27.5 & 18.2 & 8.5 \\
\hline & Median & 26.5 & 92.0 & 100.0 \\
\hline & Range & $8-100$ & $26-100$ & $81-100$ \\
\hline \multirow[t]{4}{*}{6} & $M$ & 57.5 & 86.1 & 94.2 \\
\hline & $S D$ & 29.4 & 18.1 & 7.0 \\
\hline & Median & 64.0 & 92.0 & 88.0 \\
\hline & Range & $4-100$ & $48-100$ & $86-100$ \\
\hline
\end{tabular}

Changes in level and slope between baseline and treatment conditions were analyzed statistically. There were no significant differences in the levels or slopes for any students who received the home note intervention.

CBC Group. As can be seen in Table 1, the students whose parents and teachers were involved in CBC demonstrated gains in mean accuracy scores of $144 \%$, $149 \%$, and 50\% over baseline. All three students raised their math performance from a failing level to a range comparable with that of peers who were considered by their teachers to be good math students.

Math accuracy and completion data for $\mathrm{CBC}$ students are presented in Figure 2. Baseline data for these students were variable, as expected. Consultation and the home note resulted in stable performance for all three students, with $80 \%$ $93 \%$ of all data points falling within $15 \%$ of their respective mean scores. Further support for the increased stability of the data from baseline to treatment conditions is provided by the marked reduction in the standard deviation scores from baseline to treatment for all three students. Finally, Students 4 and 5 showed little to no overlap between data points across baseline and treatment conditions. 

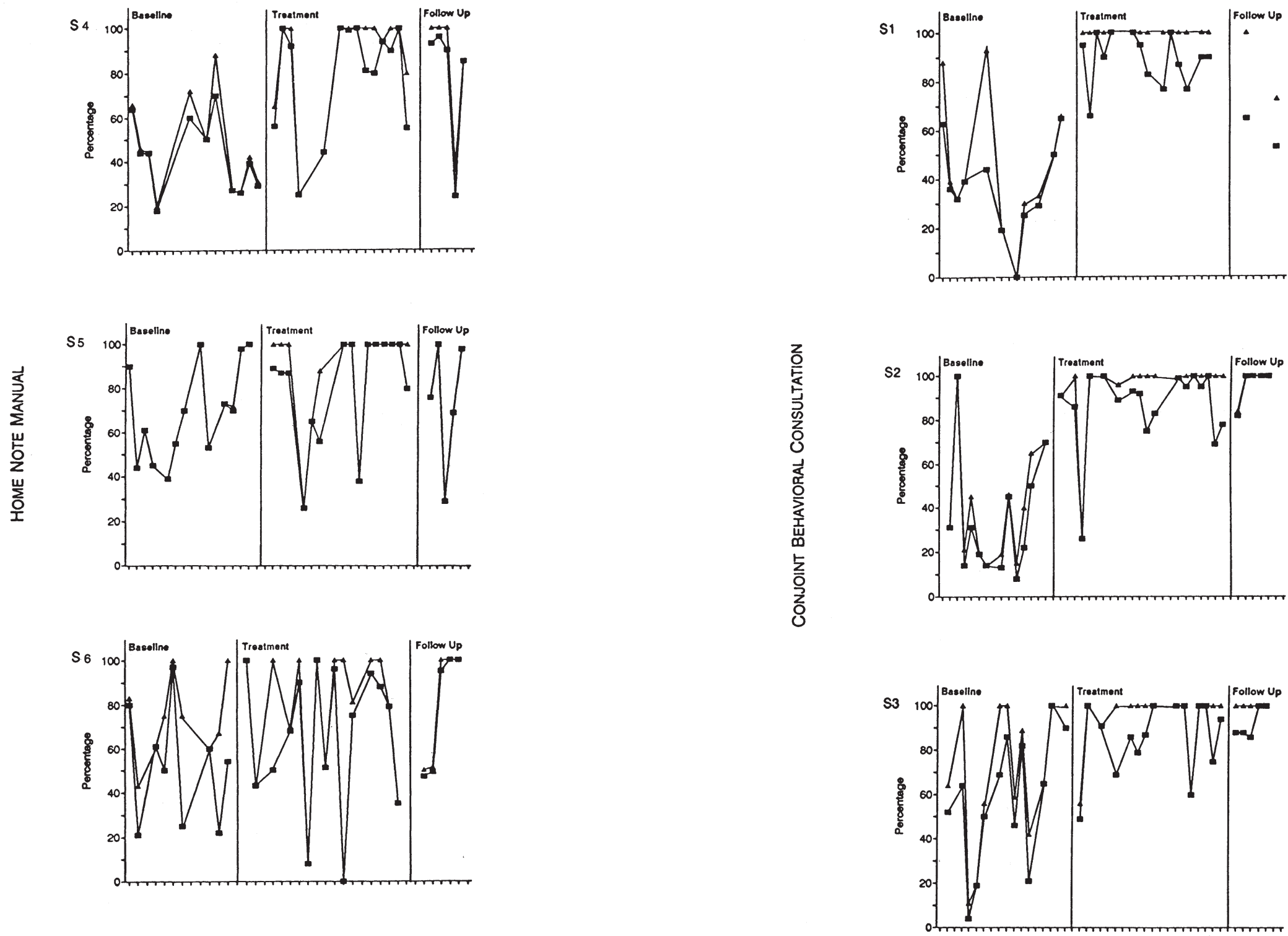

Day

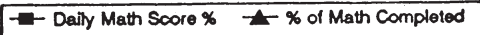

Figure 1. Math accuracy and completion data for students who received the home note intervention only.

Figure 2. Math accuracy and completion data for $\mathrm{CBC}$ students. 
There were no significant changes in slope for any CBC student. All these students, however, demonstrated a significant change $(p<.05)$ between baseline and treatment levels. This finding, in combination with visual analysis of the data in Figure 2, suggests a significant improvement in all three students' levels of work accuracy.

\section{Math Completion}

Math completion rates for all students are presented in Table 2. Brief descriptions of the findings follow.

Home Note Intervention. Mean completion scores increased for all students in the home-note-only group. Gains in mean scores of $99 \%, 27 \%$, and $14 \%$ are apparent for Students 1, 2, and 3, respectively. Completion data for all students were variable during baseline, and they remained variable during treatment for Students 1 and 3 (see Fig. 1). Treatment data for Student 2 were considered stable, although on three occasions his completion rate was at or below $65 \%$.

Table 2

\section{Math Completion Rates Across All Phases and Students}

\begin{tabular}{clccc}
\hline Student & & Baseline & Treatment & Follow-up \\
\hline Home note intervention & & & \\
1 & $M$ & 43.4 & 86.4 & 84.2 \\
& $S D$ & 20.7 & 24.3 & 27.7 \\
& Median & 44.0 & 100.0 & 100.0 \\
& Range & $25-88$ & $26-100$ & $36-100$ \\
2 & $M$ & 69.2 & 87.9 & 73.8 \\
& $S D$ & 22.0 & 24.4 & 29.1 \\
& Median & 70.0 & 100.0 & 76.0 \\
& Range & $39-100$ & $27-100$ & $29-100$ \\
3 & $M$ & 73.8 & 83.8 & 80.2 \\
& SD & 18.7 & 27.4 & 27.1 \\
& Median & 75.0 & 100.0 & 100.0 \\
& Range & $43-100$ & $4-100$ & $50-100$ \\
CBC group & & & \\
4 & $M$ & & & \\
& $S D$ & 44.4 & 100.0 & 86.5 \\
& Median & 28.2 & 0 & 19.1 \\
& Range & 38.0 & 100.0 & 86.5 \\
5 & $0-93$ & $100-100$ & $73-100$ \\
& SD & 40.6 & 94.8 & 96.8 \\
& Median & 26.8 & 17.9 & 7.2 \\
& Range & 35.5 & 100.0 & 100.0 \\
6 & $14-100$ & $29-100$ & $84-100$ \\
& SD & 69.2 & 96.5 & 100.0 \\
& Median & 31.9 & 11.4 & 0 \\
& Range & 65.0 & 100.0 & 100.0 \\
& & $12-100$ & $56-100$ & $100-100$ \\
\hline
\end{tabular}

As with accuracy data, work completion data were analyzed statistically Again, no significant changes in slope were evident for any students who received only the home note intervention. Only Student 2 demonstrated a significant change $(p<.05)$ in level between baseline and treatment. This finding, in combination with visual analysis of data in Figure 1, suggests a significant improvement in this student's level of work completion.

CBC Group. As depicted in Table 2, task completion improved considerably for all three CBC students. Gains in mean scores of $125 \%, 133 \%$, and $39 \%$ are evident for Students 4, 5, and 6, respectively. The math completion rates of all three students in the CBC group became high and stable following introduction of consultation and the home note procedure. No significant changes in slope were evident for any CBC students. Two of the three students (Students 4 and 5) demonstrated a significant change ( $p<.05$ or higher) in level between baseline and treatment. This finding, in combination with the data in Figure 2, indicates that significant improvements in levels of work completion by those students were observed as a function of the consultation-based intervention. It should be noted that changes in the completion rates of Student 6 approached significance $(p<$ .08 ), and that the completion rate of Student 6 at baseline was also the highest of the three CBC students.

\section{Maintenance}

All students in the home note group showed slight to moderate decreases in completion rates from treatment to follow-up, and two of the three showed decreases in accuracy. More importantly, as can be seen in Figure 1, the students who received only the home note intervention continued their variable performance at a 7-week follow-up, suggesting no ongoing effect of the intervention (see especially Students 2 and 3 )

Two of three CBC students (Students 5 and 6) increased their mean accuracy and completion levels at the 7 -week follow-up, neither scoring below $81 \%$ on any of the 5 days of follow-up. These data were confirmed by the teachers, who noted generally good maintenance of treatment gains, even after the interventions were withdrawn. The mother of Student 6 reported that her son indicated to her a desire to continue to do well in math, even in the absence of external rewards. Student 4 did not maintain her gains at follow-up, despite her teacher's report that she had performed well up until then. An average math score of $92.9 \%$ was reported on selected tests and daily work that had been monitored by her teacher for the period encompassing treatment, intervening weeks, and follow-up.

\section{Treatment Integrity}

Home Note Intervention Group. The integrity with which parents and teachers implemented the home note intervention was evaluated with self-report mea- 
sures (Gresham, 1989). The teachers in the home-note-only group report adhering to $78 \%-100 \%$ of the treatment objectives, with a mean of $90 \%$. Parents compliance with intervention components was lower, with a mean of $77 \%$. For parents in the home-note-only group, notes failed to be returned at least once by all three parents. One parent failed to return notes more than $60 \%$ of the time despite her stated good intentions. Tangible or activity rewards were reportedly provided for $66 \%$ of occasions on which the criteria were met. On other occasions, either verbal praise alone or long-term rewards were employed, which appeared to be provided ineffectively in some cases. For example, for Student 3 an ice-cream cone was promised after 5 days on which criteria were met. However, because of the parent's inconsistency in returning the note and the child's inconsistent math performance, only one ice-cream cone was provided over the 5 weeks of intervention, and it took the child an entire month to earn it. In another case, the parent reported at the end of the treatment period that she "did not believe" in the behavioral procedures used in the home note intervention, and provided only verbal consequences when her child met or did not meet daily criteria. No negative consequences were reported by any parents in the homenote-only group.

CBC Group. The teachers in CBC reported adhering to $84 \%-97 \%$ of treatment objectives, with a mean of $91 \%$. The parents reported an average compliance rate of $94 \%$, as assessed by self-report on the daily home notes. Over the course of the intervention (4-5 weeks), failure to return the note occurred only once for $\mathrm{CBC}$ parents. These parents appeared to be using more powerful rewards more consistently than the parents in the home-note-only group. Although the rewards provided by both groups of parents seemed appropriate, $\mathrm{CBC}$ parents provided slightly more tangible or activity rewards (vs. social rewards) than did parents in the home note group. With the exception of the first 3 days of treatment for Student 5 (on which the parent misunderstood the criterion for the reward), the $\mathrm{CBC}$ parents reported providing tangible or activity rewards (e.g., friend over, money, grab-bag prizes) either alone or in combination with verbal praise every time the criterion was met.

Consultation Integrity. Audiotapes of the CBC interviews were assessed by two independent coders for adherence to standard procedures. Across all interviews, $91 \%$ of the $\mathrm{CBC}$ objectives were met. Interrater (point-by-point) agreement between the two coders was .84 . The reliability coefficient was .43 when corrected for chance agreement by the kappa coefficient. ${ }^{2}$ In general, satisfactory adherence to the $\mathrm{CBC}$ model is suggested.

${ }^{2}$ In very skewed distributions (e.g., high concordance among raters that a behavior does or does not occur), the kappa statistic does not accurately describe the degree of interrater agreement.

\section{Social Validity}

Social validity was assessed in two ways. Specifically, social comparison and subjective evaluation procedures were used to evaluate the clinical meaningfulness of the treatment outcomes.

Social comparison. On random treatment days when teachers collected classroom data, mean math scores for the students in the home note intervention ranged from $64 \%$ to $92 \%$. This is somewhat lower than percentages obtained for matched peers (i.e., 89\%-96\%). Mean math scores for the three CBC students ranged from $83 \%$ to $92 \%$, compared with scores of matched peers, which ranged from $73 \%$ to $89 \%$. In some cases, CBC students outperformed their matched peers during the intervention phase.

Subjective Evaluation. Parents' and teachers' responses to two items on the BIRS were evaluated to determine perceptions of the students' global performance following treatment. The items in question (i.e., "The intervention proved effective in changing the child's problem behavior" and "The intervention quickly improved the child's behavior") are rated on a 6-point scale and yielded averages of 4.3 and 4.0 (Slightly agree), respectively, for teachers in the home note group. Mean parent ratings were 5.0 and 4.7 (Agree). In contrast, the $\mathrm{CBC}$ teachers gave a rating of 6 (Strongly agree) to both social validity questions. The consultation parents' ratings averaged 5.7 and 5.0 on the two items, respectively.

\section{Treatment Acceptability}

Behavior Intervention Rating Scale (BIRS). The results of the parents' and the teachers' ratings on the BIRS (Von Brock \& Elliott, 1987) are presented in Table 3. On a scale of 1. to 6 (with 6 representing high acceptability), the teachers in the home note group provided a mean rating across items of 4.5 (Slightly agree). The highest rating was obtained on Item 5 (appropriateness of the intervention, given problem severity). The lowest ratings were provided on items assessing social comparisons (Items 18 and 22). Parents provided a mean rating across items of 4.97 (Agree) on the BIRS. The highest ratings were obtained on Items 2, 8 , and 9 (assessing appropriateness of the intervention for other problems and children, and the lack of negative side effects). The lowest-rated item by parents in the home note group (Item 17) concerned perceptions regarding the lasting improvement in the child's behavior.

The teachers in the CBC group provided an overall mean rating across BIRS items of 5.71 (Strongly agree), suggesting that the consultation intervention was perceived as highly acceptable. All 15 items on the Acceptability factor of the BIRS were given ratings of 6 by teachers in this group. The lowest-rated items 
Table 3 Mean BIRS Ratings Across Items and Subjects

\begin{tabular}{lccccc}
\hline & \multicolumn{2}{c}{ Home note group } & & \multicolumn{2}{c}{ CBC groups } \\
\cline { 2 - 3 } \cline { 5 - 6 } Item & Parents & Teachers & & Parents & Teachers \\
\cline { 6 - 7 } 1. Intervention acceptability & 5.3 & 5.0 & & 5.7 & 6.0 \\
2. Generalizability of intervention & 5.7 & 5.0 & & 5.7 & 6.0 \\
3. Intervention effectiveness & 5.0 & 4.0 & & 5.7 & 6.0 \\
4. Referral of intervention to others & 5.3 & 5.0 & & 5.7 & 6.0 \\
5. Problem warrants intervention & 5.3 & 6.0 & & 5.3 & 6.0 \\
6. Suitability of intervention & 5.3 & 5.0 & & 4.7 & 6.0 \\
7. Willingness to use again & 5.3 & 4.5 & & 5.7 & 6.0 \\
8. No negative side effects & 5.7 & 5.0 & & 5.7 & 6.0 \\
9. Appropriateness for variety of children & 5.7 & 4.5 & & 5.7 & 6.0 \\
10. Consistent with previous interventions & 5.3 & 4.5 & & 3.7 & 6.0 \\
11. Fairness of intervention & 5.3 & 5.0 & & 5.0 & 6.0 \\
12. Reasonability of intervention & 5.3 & 5.0 & & 5.0 & 6.0 \\
13. Pleased with intervention procedures & 5.3 & 4.5 & & 5.7 & 6.0 \\
14. Appropriateness of intervention & 5.0 & 5.0 & & 5.7 & 6.0 \\
15. Beneficial for child & 5.3 & 4.5 & & 6.0 & 6.0 \\
16. Produced quick improvement & 4.7 & 3.5 & & 4.7 & 6.0 \\
17. Produced lasting improvement & 3.7 & 3.5 & & 4.3 & 5.5 \\
18. Produced improvement equal to peers & 5.0 & 3.0 & & 5.0 & 5.0 \\
19. Positive change noted & 5.3 & 4.5 & & 5.3 & 6.0 \\
20. Maintenance of behavior change & 4.0 & 4.5 & & 4.3 & 5.0 \\
21. Generalization of behavior change & 4.3 & 4.5 & & 4.3 & 5.0 \\
22. Social comparability after intervention & 4.5 & 3.0 & & 3.7 & 5.5 \\
23. Behavior no longer a problem & 4.0 & 5.0 & & 4.7 & 4.5 \\
24. Other behaviors improved & 5.0 & 5.0 & & 4.3 & 4.5 \\
Average across subjects and items & 5.0 & 4.5 & & 5.1 & 5.7 \\
\hline
\end{tabular}

concerned complete elimination of the problem (Item 23) and generalization of treatment effects to other behaviors (Item 24). CBC parents also rated the procedures as generally acceptable, with a mean rating of 5.06 (Agree). The highest parent rating was obtained on Item 15 (assessing overall benefit for the student). The lowest parent ratings were obtained on items assessing previous use of the model (Item 10) and social comparability following the intervention (Item 22).

Children's Intervention Rating Profile (CIRP). The students completed the CIRP (Turco \& Elliott, 1986) to assess their acceptability of the home note intervention. On a scale of 1-5 (with 5 indicating high acceptability), a mean item rating of 4.3 (Moderately acceptable) was obtained across students who received the home note intervention only. The students provided ratings of 5 to several items (e.g., "I liked the plan"; "The plan helped me do better in school"). The lowest mean rating was obtained on an item assessing fairness of the plan, although this was rated as moderately acceptable.

For CBC students, a mean item rating of 4.67 (Highly acceptable) was ob- tained. These students also provided ratings of 5 to several items. The lowest mean rating was obtained on an item assessing more appropriate ways to handle the problem.

\section{DISCUSSION}

This article has illustrated the use of two empirically based interventions in the treatment of academic performance problems, presented in the contex of carefully controlled case studies. The data suggest that home-school communication systems such as home notes, when used alone, provide equivocal treatment effects. Use of the home note and self-instruction manual without consultation brought the math scores of two of the three students into a range acceptable to their classroom teacher; however, the performance of all the students continued to be variable. Likewise, the home note procedure failed to produce statistically significant changes in levels of work accuracy for every student, and produced nonsignificant results for the levels of work completion of two of the three students. When implemented in the context of $\mathrm{CBC}$, the home note intervention was effective at bringing the math performance of students into a range comparable with that of others in the classroom who were considered to be good in math. All students whose parents and teacher were involved in $\mathrm{CBC}$ demonstrated statistically significant gains in work accuracy, and two of the three showed significant gains in work completion. The CBC model also appeared to be strong in terms of maintenance of treatment gains and in consumer acceptability.

In addition to general performance gains, it is important to examine the variability of students' academic performances across baseline and treatment For all students, math scores and completion rates were variable at baseline. For students who received only the home note intervention, performance variability increased during the treatment phase for both accuracy and completion. Performance variability increased even further at follow-up. For CBC students, variability declined markedly during treatment. For two of the three $\mathrm{CBC}$ students, variability declined even further during follow-up. This decrease in performance variability is important, as inconsistent performance was a major complaint of the students' teachers prior to implementation of the interventions.

These data support the contention of previous authors (Blechman, Kotanchik, \& Taylor, 1981; Blechman, Taylor, \& Schrader, 1981; Karraker, 1972 that for students with severe or intractable academic performance problems, more intensive consultation appears to be needed to maximize treatment gains. The present data suggest that, for students with a history of academic performance problems who have not responded to more traditional intervention strategies, parent-teacher consultation may be essential to improve academic 
performance. This does not appear to be due simply to the provision of information, as a home note manual was distributed to all parents and teachers that provided the same information as the consultation model with regard to setting up home rewards for school performance. It is more likely that the improvement factors were embedded in the nature of the relationship that developed among participants in the consultation process, although this was not tested directly.

Consultees, particularly teachers, expressed great satisfaction with the process and outcomes of consultation. Satisfaction ratings of CBC teachers were higher than the ratings of the other teachers. All participants may have felt more accountable in fulfilling their agreements because they were to be discussed in the TEI.

\section{Strengths of the Case Studies}

These case studies provide support for the use of CBC with students who experience academic performance problems. They illustrate procedures by which carefully controlled case study research can be conducted in natural settings and support the emphasis of integrating a scientist-practitioner model into school psychology practice. The type of data collected was similar to that typically recorded by most classroom teachers. The teachers appeared to value the additional input provided by the consultation model despite the fact that it required a moderate time commitment. The fact that treatment gains were maintained for most of the CBC students added to the perceived value of the model. Of particular importance is the fact that procedures were used to strengthen the intervention case studies methodologically and to minimize various threats to internal validity. These include (a) the use of multiple objective measures, (b) repeated assessment across baseline, treatment, and follow-up conditions, (c) active manipulation of the independent variable, (d) standardization of assessment and treatment, (e) checks on treatment integrity, (f) social validity and treatment acceptability assessment, (g) a formal A-B design with followup, (h) time series analyses, and (i) replication across subjects (Kratochwill, 1985).

Problem identification and analysis of academic problems in these case studies revealed meaningful procedures by which to target performance problems within a consultative framework. Furthermore, evaluation of treatment gains was strengthened by the use of meaningful and relevant curriculumbased classroom measures (Salvia \& Hughes, 1990). Specifically, daily assignments were used as measures of math performance, which are the same curriculum-based measures as those used by teachers to assess students' math achievement

The present case studies also have the advantage of addressing issues of treatment integrity, social validation, and consumer satisfaction. These variables are infrequently measured in natural settings or in intervention/consultation studies (Witt \& Elliott, 1985). However, they are important for making consultation research relevant and meaningful to practitioners (Gresham, 1989, 1991)

\section{Limitations of the Case studies}

Although the $\mathrm{A}-\mathrm{B}$ design with replication is considered to be acceptable for use in applied settings (Harris \& Jenson, 1985), it does not constitute a true experiment. Importantly, historical threats cannot be systematically ruled out, which limits the ability to draw inferences about functional relationships between the intervention and behavioral changes.

Systematic increases in baseline without the added experimental control afforded by a design such as the multiple baseline leave open the possibility that historical factors other than the treatments themselves were responsible for the observed changes during treatment. An interesting finding was the increasing trend in baseline data that was apparent for all CBC students and one home note student (Student 2). This trend began 4-5 days prior to the implementation of the intervention. Two events occurred at approximately the same time. First, all students were told that their work was being collected and looked at by the school psychologist. Second, PIIs were conducted with parents and teachers of all CBC students. Because only one of the three students in the home note group appeared to change his behavior in relation to being told that his work was being evaluated, something unique to the PIIs may have had a greater influence than the student contacts in producing noticeable changes in students' performances. In other words, simply initiating consultation between the school psychologist, parents, and teachers may have had positive effects on students' behaviors even without the implementation of a home note intervention. Previous research has shown that more inclusive forms of behavioral consultation lead to greater treatment effects than procedures that include only one or two stages (Fuchs \& Fuchs, 1989). Therefore, it is not known if these positive changes would have been maintained in the absence of a behavioral intervention aimed at increasing work completion and accuracy. However, it is likely that the parents and teachers in the present $\mathrm{CBC}$ cases engaged in certain behaviors as a function of their initial involvement in $\mathrm{CBC}$ that produced concomitant changes in students' behaviors prior to the implementation of the home note intervention. This represents a historical event influencing all CBC students and limits the ability to draw inferences about the functional relationship between the intervention and behavioral changes.

One way of supporting the conclusion that the treatments were responsible for performance gains is by evaluating the amount of improvement in academic performance as it relates to the degree to which the treatments were implemented by the students' parents. Figure 3 depicts a scatterplot in which the degree of improvement is plotted against the degree of implementation for each 


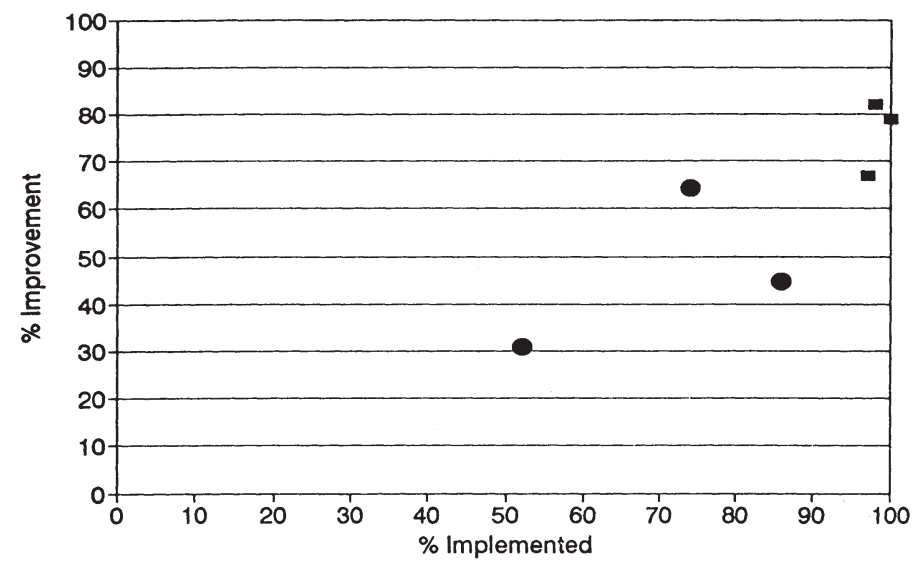

- $\mathrm{CBC} \bullet \mathrm{HNM}$

Figure 3. Scatterplot depicting degree of student improvement in relation to treatment integrity.

of the six students. The data clearly suggest that higher rates of improvement are associated with higher percentages of treatment implementation. These data support the conclusion that performance gains were in fact related to the interventions. Parenthetically, CBC students showed much higher rates of both treatment integrity and academic improvement than did students in the home note intervention.

In analyzing the data in Figure 3, one might speculate that consultation is useful in increasing participants' investment and accountability for the outcome of the intervention. Parents in both groups used ideas for home reinforcers provided in the manual; however, the parents in the CBC group appeared to use more powerful reinforcers more consistently than did the other parents. Review of the selfmonitoring checklists indicated that $\mathrm{CBC}$ parents were more compliant than the other parents in checking the home note, praising the child for improved performance, and providing agreed-upon reinforcers. In addition, rates of students' compliance with "process" behaviors (i.e., those intended to assist them in meeting their daily performance goal) were generally better for $\mathrm{CBC}$ students than for the others. The latter result may reflect a greater commitment on the part of $\mathrm{CBC}$ parents to remind and reinforce students for appropriate organizational behaviors, as was discussed in the context of the CBC interviews.

A further limitation of the case studies is the lack of control over math instruction and assignments received by students over the course of the interven- tion. Specifically, information regarding type and amount of math instruction taking place is unavailable. Instrumentation presents a possible threat to the validity of the case studies in that the students were not engaged in identical math tasks across assessment occasions, and variability in the difficulty level of the assignments was not controlled. It is apparent in Figures 1 and 2 that there may have been common days when several students performed similarly (i.e., days when data across several students dropped well below their respective means). Although not all students were in the same classroom and they did not all receive identical math assignments, it is that aspects of the math tasks or some common event influenced their performance.

The generalizability of the conclusions also may be limited by selection and attentional factors. The school in which the case studies were conducted is generally "high-functioning" and espouses a commitment to parent involvement. All parents and teachers agreed to participate, and most were initially enthusiastic. The same person served as consultant in all CBC cases, affording no control over therapist effects. Finally, the A-B case study design does not control for time and attention factors that may have contributed to treatment outcomes for CBC students.

\section{Implications for Practice}

These case studies suggest several implications for practicing school psychologists. The results support previous research on the usefulness of involving parents in their children's educational efforts (Epstein, 1988). For children with relatively severe and intractable academic performance problems, the use of a structured parent-teacher behavioral consultation model may strengthen existing home-school communication techniques. Likewise, it reinforces Kramer's (1990) observation concerning the need to supplement manual-based interventions with a structured and individualized approach such as consultation. Consultation may increase the accountability of all participants and allow for early clarification and modification of the treatment regimen.

It is recognized that the multitude of measures used in the case studies may appear overwhelming and impractical for school psychologists conducting interventions and consultation cases in everyday practice. For example, measures of effectiveness, social validity, treatment acceptability, and treatment integrity were collected. Several of these were used and reported here for purposes of illustration, and although we believe that they are all important components of outcome, it is possible to conduct consultation and intervention cases and draw meaningful conclusions with fewer measures. When determining which measures to include, there are two basic considerations: relevance and cost-benefit ratio.

When limited resources are available for evaluating intervention and consultation outcomes, an essential criterion is that selected measures be relevant. Therefore, the purpose of the intervention must be clear in order to collect out- 
come data directly related to the referral questions. For example, if work completion is the target concern, measuring outcome can be as simple as conducting simple frequency counts or looking at a teacher's record book. If increasing a child's compliance with initial parent and teacher commands is the primary treatment objective, however, data on academic competence is not necessary to determine intervention outcomes. The cost-benefit ratio pertains to the amount and quality of information gleaned at a given cost. For example, a wealth of important consumer satisfaction data may be obtained by using a simple, inexpensive rating scale such as the BIRS, which assesses both intervention acceptability and subjective evaluations of treatment effectiveness. Likewise, social comparison data can be collected easily during observation of a target student, or obtained by requesting random scores of classmates from a teacher's existing records. In contrast, the administration of lengthy standardized test batteries is high in cost and may yield little functional information regarding behavioral concerns or responsiveness to an intervention. In this set of case studies, checks on the integrity of the consultation procedures were conducted by audiotape analysis. This level of evaluation is costly, and will not always be necessary. In general, the minimum procedures for evaluating consultation and intervention outcomes should include direct observations conducted repeatedly, behavior-specific rating scales, and self-reports of treatment integrity. When possible, consumer satisfaction and social validity data are also helpful.

\section{Research Needs}

Research is needed to further document the efficacy of $\mathrm{CBC}$ as an effective model of home-school collaboration in the remediation of academic performance deficits. Specifically, it would be useful to replicate the present results with diverse populations of students (e.g., students with learning disabilities, behavioral disorders, and communication disorders) and diverse target behaviors (e.g., noncompliance, disruptive classroom behaviors, and academic skills deficits). Incorporating methodological changes to address the limitations of the present case studies will be especially important. This includes implementation of a research design that (a) allows for stricter experimental control (e.g., multiple baseline or reversal design); (b) controls for time and attention factors; (c) systematically attends to social comparison issues; and (d) assesses long-term follow-up. In addition, a larger student sample across varying demographic and socioeconomic conditions would enhance confidence in $\mathrm{CBC}$ as an effective intervention model.

As time limitations are an ever-present reality in the schools, it may be important to streamline the consultation process by identifying the components of $\mathrm{CBC}$ necessary to achieve treatment gains. The behavioral consultation model provides some duplication across Problem Identification and Problem Analysis Interviews, in particular. For problems that are relatively clearly defined, an ef- fort to reduce duplication of information may make consultation more efficient for use in school settings. In addition, the home note, accompanied by a self-instruction manual, demonstrated some effectiveness in enhancing academic performance. Future research may determine that multiple levels of intervention may be appropriate, depending on the extent of performance deficits.

The present case studies suggest that factors embedded in the consultation relationship may have been responsible for observed differences. Factors such as opportunities to clarify intervention components and troubleshoot problems early in treatment, the added accountability built into CBC and the development of a collegial relationship among all participants may have added to the efficacy of the CBC model. Future research should systematically explore the manner in which relationship factors (e.g., consultant and consultee characteristics and communication styles) contribute to consultation effectiveness.

The present $\mathrm{CBC}$ cases investigated the model with parents involved actively in all interviews. It is generally acknowledged, however, that some parents may be unwilling or unable to come to the school for consultation sessions. It is possible that some intermediate level of parent involvement may prove advantageous for these parents. Future research should examine alternative parent roles and levels of participation in the consultation process.

One anticipated outcome of consultation is the improvement of consultees' skills such that they may be applied to solve similar problems in the future. Likewise, a goal of CBC is to enhance the home-school relationship and to encourage continued collaboration between parents and teachers. The present case studies do not adequately explore these consultation objectives. Analysis of the generalization of the techniques acquired through the consultation might be a focus of future research

\section{ACKNOWLEDGMENTS}

The authors express gratitude to Drs. William Jenson, Howard Sloane, K. Richard Young, Elaine Clark, and John Kircher for their assistance in this research. They are also very grateful to Thomas R. Kratochwill for his helpful comments on earlier drafts of this manuscript, and to Ryan Gothard for his assistance with graphics.

\section{REFERENCES}

Bergan, J. R., \& Kratochwill, T. R. (1990). Behavioral consultation in applied settings. New York: Plenum.

Blechman, E. A., Kotanchik, N. L., \& Taylor, C. J. (1981). Families and schools together: An early behavioral intervention with high-risk children. Behavior Therapy, 12, 308-319.

Blechman, E. A., Taylor, C. J., \& Schrader, S. M. (1981). Family problem solving versus home notes as early intervention with high-risk children. Journal of Consulting and Clinical Psychology, 49, 919-926. 
Budd, K. S., Leibowitz, M., Riner, L. S., Mindell, C., \& Goldfarb, A. L. (1981). Home-based treatment of severe disruptive behaviors: A reinforcement package of preschool and kindergarten children. Behavior Modification, 5, 273-298.

Crosbie, J., \& Sharpley, C. F. (1991). DMITSA 2.0: A statistical program for analysing data from interrupted time series. Author: Victoria, Australia.

Elliott, S. N. (1986). Children's ratings of the acceptability of classroom interventions for misbehavior: Findings and methodological considerations. Journal of School Psychology, 24, 23-35.

Elliott, S. N., \& Von Brock Treuting, M. (1991). The Behavior Intervention Rating Scale: Development and validation of a pretreatment acceptability and effectiveness measure. Journal of School Psychology, 29, 43-51.

Epstein, J. L. (1988). How do we improve programs for parent involvement? Educational Horizons, 66, 57-59.

Fairchild, T. N. (1976). Home-school token economies: Bridging the communication gap Psychology in the Schools, 13, 463-467.

Fuchs, D., \& Fuchs, L. S. (1989). Exploring effective and efficient prereferral interventions: A component of behavioral consultation. School psycho logy Review, 18, 260-283.

Gresham, F. M. (1989). Assessment of treatment integrity in school consultation and prereferral intervention. School Psychology Review, 18, 37-50.

Gresham, F. J. (1991). Moving beyond statistical significance in reporting consultation outcome research. Journal of Educational and Psychological Consultation, 2, 1-13.

Harris, F. N., \& Jenson, W. R. (1985). Comparison of multiple baseline across person designs and A-B designs with replication: Issues and confusion. Behavioral Assessment 7, 121-127

Karraker, R. J. (1972). Increasing academic performance through home-managed contingency programs. Journal of School Psychology, 10, 173-179.

Kaufman, A. S., \& Kaufman, N. L. (1990). Kaufman Brief Intelligence Test. Circle Pines, MN: American Guidance Service.

Kazdin, A. E. (1977). Assessing the clinical or applied importance of behavior change through social validation. Behavior Modification, 1, 427-451.

Kazdin, A. E., French, N. H., \& Sherik, R. B. (1981). Acceptability of alternative treatments for children: Evaluations by inpatient children, parents and staff. Journal of Consulting and Clinical Psychology, 49, 900-907.

Kelley, M. L., \& Carper, L. B. (1988). Home-based reinforcement procedures. In J. C. Witt, S. N. Elliott, \& F. M. Gresham (Eds.), Handbook of behavior therapy in education (pp. 419-438). New York: Plenum.

Kramer, J. J. (1990). Training parents as behavior change agents: Successes, failures, and suggestions for school psychologists. In T. B. Gutkin \& C. R. Reynolds (Eds.), The handbook of school psychology (2nd ed., pp. 683-701). New York: Wiley.

Kratochwill, T. R. (1985). Case study research in school psychology. School Psychology Review, 14, 204-215.

Kratochwill, T. R. \& Bergan, J. R. (1990). Behavioral consultation in applied settings: An individual guide. New York: Plenum.

Kratochwill, T. R., Sheridan, S. M., Rotto, P. C., \& Salmon, D. (1991). Preparation of school psychologists to serve as consultants for teachers of emotionally disturbed children. School Psychology Review, 20, 530-550.
Kratochwill, T. R., VanSomeren, K. R., \& Sheridan, S. M. (1989). Training behavioral consultants: A competency-based model to teach interview skills. Professional School Psychology, 4, 41-58.

Lahey, B. B., Gendrich, J. G., Gendrich, S. I., Schnelle, J. F., Gant, D. S., \& McNees, M. P. (1977). An evaluation of daily report cards with minimal teacher and parent contacts as an effective method of classroom intervention. Behavior Modification, 1, 381-394.

McGinnis, E., \& Goldstein, A. P. (1984). Skillstreaming the elementary school child: A guide for teaching prosocial skills. Champaign, IL: Research Press.

Olympia, D. E., Jenson, W. R., \& Neville, M. R. (1990). Do it yourself homework manual. A sanity saver for parents. (Available from W. Jenson, Dept. of Educational Psychology, $327 \mathrm{MBH}$, University of Utah, SLC, UT 84112.

Salvia, J., \& Hughes, J. (1990). Curriculum-based assessment: Testing what is taught. New York: Macmillan.

Schumaker, J. B., Hovell, M. F., \& Sherman, J. A. (1977). An analysis of daily report cards and parent-managed privileges in the improvement of adolescents' classroom performance. Journal of Applied Behavior Analysis, 10, 449-464.

Sheridan, S. M. (1992). Consultant and client outcomes of competency-based behavioral consultation training. School Psychology Quarterly, 7, 245-270.

Sheridan, S. M., \& Colton, D. L. (1994). Conjoint behavioral consultation: A review and case study. Journal of Educational and Psychological Consultation, 5, 211-228.

Sheridan, S. M., \& Kratochwill, T. R. (1992). Behavioral parent-teacher consultation: Conceptual and research considerations. Journal of School Psychology, 30, 117-139.

Sheridan, S. M., Kratochwill, T. R., \& Bergan, J. R. (in press). Conjoint behavioral consultation: A procedural manual. New York: Plenum.

Sheridan, S. M., Kratochwill, T. R., \& Elliott, S. N. (1990). Behavioral consultation with parents and teachers: Delivering treatment for socially withdrawn children at home and at school. School Psychology Review, 19, 33-52.

Tawney, J. W., \& Gast, D. L. (1984). Single-subject research in special education. Columbus, OH: Charles E. Merrill.

Turco, T. L., \& Elliott, S. N. (1986). Assessment of students' acceptability ratings of teacherinitiated interventions for classroom misbehavior. Journal of School Psychology, 24, 277-283.

Von Brock, M. B., \& Elliott, S. N. (1987). Influence of treatment effectiveness information on the acceptability of classroom interventions. Journal of School Psychology, 25 $131-144$

Witt, J. C., \& Elliott, S. N. (1985). Acceptability of classroom management strategies. In T. R. Kratochwill (Ed.), Advances in school psychology (Vol. 4, pp. 251-288). Hillsdale, NJ: Lawrence Erlbaum.

Woodcock, R. W., \& Mather, N. (1990). Woodcock-Johnson Psychoeducational Battery-Revised. Allen, TX: DLM Teaching Resources. 